\title{
Estudio preliminar del origen del agua del Valle de Uspallata y de su contribución al río Mendoza mediante técnicas isotópicas e hidroquímicas. Mendoza, Argentina
}

\author{
Sandra P. Ibáñez ${ }^{(1)}$, Belén Lana ${ }^{(2)}$, Marisol Manzano(3), Susana Rovira(1), Enrique Montero ${ }^{(4)}$, Natalia \\ Salvi ${ }^{(5)}$, Héctor Segal ${ }^{(4)}$, PablaTognoli ${ }^{(4)}$, Pablo Grizas ${ }^{(4)}$, Leandro Salvioli(5) ${ }^{(2)}$ Diego Márquez ${ }^{(5)}$, Juan \\ Pina $^{(4)}$ y Daniel Cicerone ${ }^{(6)}$
}

(1) Facultad de Ingeniería de la UNCuyo (FIUNCuyo). Centro Universitario, M5502JMA Mendoza, Argentina. sandra.ibanez@ingenieria.uncu.edu.ar; susana.rovira@ingenieria.uncu.edu.ar (2) Instituto Argentino de Nivología, Glaciología y Cs. Ambientales (IANIGLA). Av. Ruiz Leal s/n, (5500) Mendoza, Argentina. blana@mendoza-conicet.gov.ar

(3) Departamento de Ingeniería Minera y Civil, Universidad Politécnica de Cartagena. $\mathrm{P}^{\circ}$ de Alfonso XIII, 52, Cartagena. España. marisol.manzano@upct.es

(4) Dpto. General de Irrigación (DGI), Av. España \& Barcala, M5500 Mendoza. Argentina. jpina@agua.gob.ar, hsegal@agua.gob.ar, emontero@agua.gob.ar, ptognoli@agua.gob.ar

(5) Instituto Nacional del Agua (INA), Av. Belgrano 210, M5500CFA Mendoza, Argentina. natisalvi77@gmail.com; Isalvioli@ hotmail.com; diegormarquez@ hotmail.com

(6) Comisión de Energía Atómica (CNEA). Av. del Libertador 8250, C1429BNP, CABA, Argentina. cicerone@cnea.gov.ar

\section{RESUMEN}

El Valle de Uspallata está situado en la Provincia de Mendoza (Argentina), a unos $1900 \mathrm{~m}$ snm. Con dirección norte-sur y unos $20 \mathrm{~km}$ de longitud, constituye un valle intermontano localizado entre la Precordillera y la Cordillera Frontal. En su extremo sur descarga al río Mendoza, principal recurso hídrico que abastece al oasis socio-productivo de mayor importancia de la provincia de Mendoza. Desde hace dos décadas se observa un crecimiento urbano significativo en la zona sur del valle, con desarrollos de actividades agrícolas, turísticas y emprendimientos inmobiliarios, sin planes de ordenamiento adecuados tendentes a preservar la calidad del recurso hídrico. En este contexto, el objetivo del trabajo es establecer un modelo conceptual sobre la procedencia del agua del acuífero a lo largo del valle y sobre la magnitud del aporte de esta cuenca al río Mendoza. El propósito último es generar información de base que contribuya a la toma de decisión de los organismos de gestión en referencia a la adecuada gestión del recurso agua y el ordenamiento territorial. Para conocer las características y la procedencia del agua del acuífero del valle se han aplicado técnicas isotópicas e hidroquímicas. Además de recopilar datos de los pocos estudios anteriores existentes, se realizaron dos campañas de monitoreo en las cuales se midieron parámetros físico-químicos e isótopos estables en 39 sitios, comprendiendo pozos, manantiales, arroyos y el río Mendoza. Los balances isotópicos preliminares indican que alrededor del $70 \%$ del agua subterránea del valle procede de Cordillera Frontal. Además, la interpretación integrada de datos nuevos e información geológica y geoeléctrica previa permitió establecer un modelo conceptual preliminar que diferencia tres sectores respecto a la procedencia del agua en la Cordillera Frontal. El trabajo forma parte del proyecto internacional ARG/7/008 “Uso de Técnicas isotópicas para la mejora de la gestión del recurso hídrico subterráneo", financiado por el Organismo Internacional de Energía Atómica.

Palabras clave: Balance de masas, Hidroquímica, Isótopos estables, Valle de Uspallata.

\section{Preliminary study of water origin in the Uspallata valley and its contribution to the Mendoza river with isotopic and hydrochemical techniques. Mendoza, Argentina}




\section{ABSTRACT}

The Uspallata Valley is located in Mendoza Province (Argentina), at an average altitude of 1,900 $\mathrm{m}$ asl. With a north-south direction and a length of around $20 \mathrm{~km}$, it is an intermountain valley placed between the Pre-cordiIlera (to the east) and the Frontal Cordillera (to the west). The valley discharges to the Mendoza River, the main Andean course that irrigates the largest socio-productive oasis in Mendoza Province. For the last 20 years there has been a significant increase in urban growth and a disordered development of agricultural, touristic, and real estate activities in the southern part of the valley. The objective of this study is to use isotopic and hydrochemical techniques to develop a geohydrological conceptual model, in order to contribute to decision making processes of the provincial management that deals with water and land-use planning. Hydrochemical and isotopic techniques have been used to understand groundwater characteristics and provenance along the valley. Beyond compiling the scarce previous existing data, two monitoring surveys were carried out where physical-chemical parameters and stable isotopes were measured in 39 sites (wells, springs, creeks, and the Mendoza River). The preliminary isotopic balances performed suggest that around $70 \%$ of the groundwater comes from the Cordillera Frontal. Moreover, the integrated analysis of new data and existing geological and geoelectric information has allowed the development of a preliminary conceptual model identifying three zones relating to the origin of the water along the Frontal Cordillera. The study is part of the project "Use of isotopic techniques for improving the management of groundwater resources" ARG/7/008, which is funded by the International Atomic Energy.

Keywords: mass balance, hydrochemistry, stable isotopes, Valle de Uspallata.

\section{Introducción}

El Valle de Uspallata es un valle intermontano ubicado $90 \mathrm{~km}$ al noroeste de la capital de la Provincia de Mendoza y a $1900 \mathrm{~m}$ snm. En la actualidad el incremento de las actividades turísticas de alta montaña y los emprendimientos inmobiliarios de barrios privados han generado un crecimiento desordenado en la Villa, que pasó de 2932 habitantes en 1991 a más de 9654 en el año 2010 (INDEC, 1991, 2010). Contrariamente, las redes de agua potable y cloaca no tuvieron el mismo desarrollo, por lo que hay sectores que no poseen estos servicios. Para suplir estas necesidades se están realizando pozos clandestinos para el abastecimiento poblacional y, en sus inmediaciones, pozos sépticos para desechar las aguas servidas. Existen pocos estudios sobre la cuenca hidrogeológica del Valle de Uspallata. Los mismos se remontan a las décadas de 1980 y 1990 y constituyen la base del presente trabajo. Este pretende establecer un modelo conceptual sobre la procedencia del agua del acuífero a lo largo del valle y sobre la aportación global al río Mendoza, objetivos fundamentales para establecer una gestión ordenada del recurso.

\section{Zona de estudio}

El Valle de Uspallata se extiende de norte a sur entre la Cordillera Frontal al oeste y la Precordillera al este (Fig. 1). Se trata de una depresión tectónica que fue rellenada por sedimentos de ambas vertientes, originando la denominada cuenca hidrogeológica del Valle de Uspallata. Esta se asienta sobre un basamento de sedimentitas neógenas, tiene espesores variables entre $175 \mathrm{~m}$ al norte a $25 \mathrm{~m}$ al sur y pendiente descendente de norte a sur. En la cuenca se han desarrollado acuíferos libres en sedimentos de granulometría gruesa a mediana. La Cordillera Frontal está representada en esta zona por la Cordillera del Tigre, que alcanza cotas superiores a los $5500 \mathrm{~m}$ snm. Está constituida dominantemente por rocas ígneas (volcánicas y plutónicas) y piroclásticas del Grupo Choiyoi (Caminos, 1979) de edad permo-triásica. Existen también rocas más antiguas, sedimentarias en su mayor parte, que han sido intruidas por las rocas plutónicas o sirven de base a las volcánicas. La Precordillera está representada por la Sierra de Uspallata y otros cordones menores, con cotas máximas que superan los $3000 \mathrm{~m}$ snm. Está constituida principalmente por rocas sedimentarias del Paleozoico y por rocas sedimentarias y volcánicas delTriásico (Vaca, 1985).

Por su ubicación geográfica, el Valle de Uspallata presenta un gran dinamismo atmosférico. Entre los meses de mayo y octubre se producen nevadas provenientes del Océano Pacífico y en verano se producen Iluvias provenientes del Océano Atlántico (Capitanelli, 1972). La precipitación líquida media anual es menor a $150 \mathrm{~mm}$.año-1 (1961-1990) y la evaporación potencial media anual supera los $1300 \mathrm{~mm} \cdot \mathrm{año}^{-1}$ (1961-1990) (Salomón et al., 2005).

La rigurosidad del clima reduce las posibilidades productivas agropecuarias a la actividad forestal, la producción de papa y semilla de papa y cierta ganadería de cría. La producción agrícola se desarrolla bajo riego utilizando el agua superficial de los cursos de agua permanente del valle, los arroyos San Alberto, Tambillo y Chiquero, que poseen régimen nival con crecidas en primavera-verano. Otro curso de agua presente es el A. Uspallata el cual atraviesa el valle de norte a sur. En su recorrido el arroyo se comporta como el principal colector de la cuenca, evidenciado por el aumento de su caudal en el sentido de dirección de su flujo (Vaca, 1985, 1993; Gianni y Vaca, 1994). 


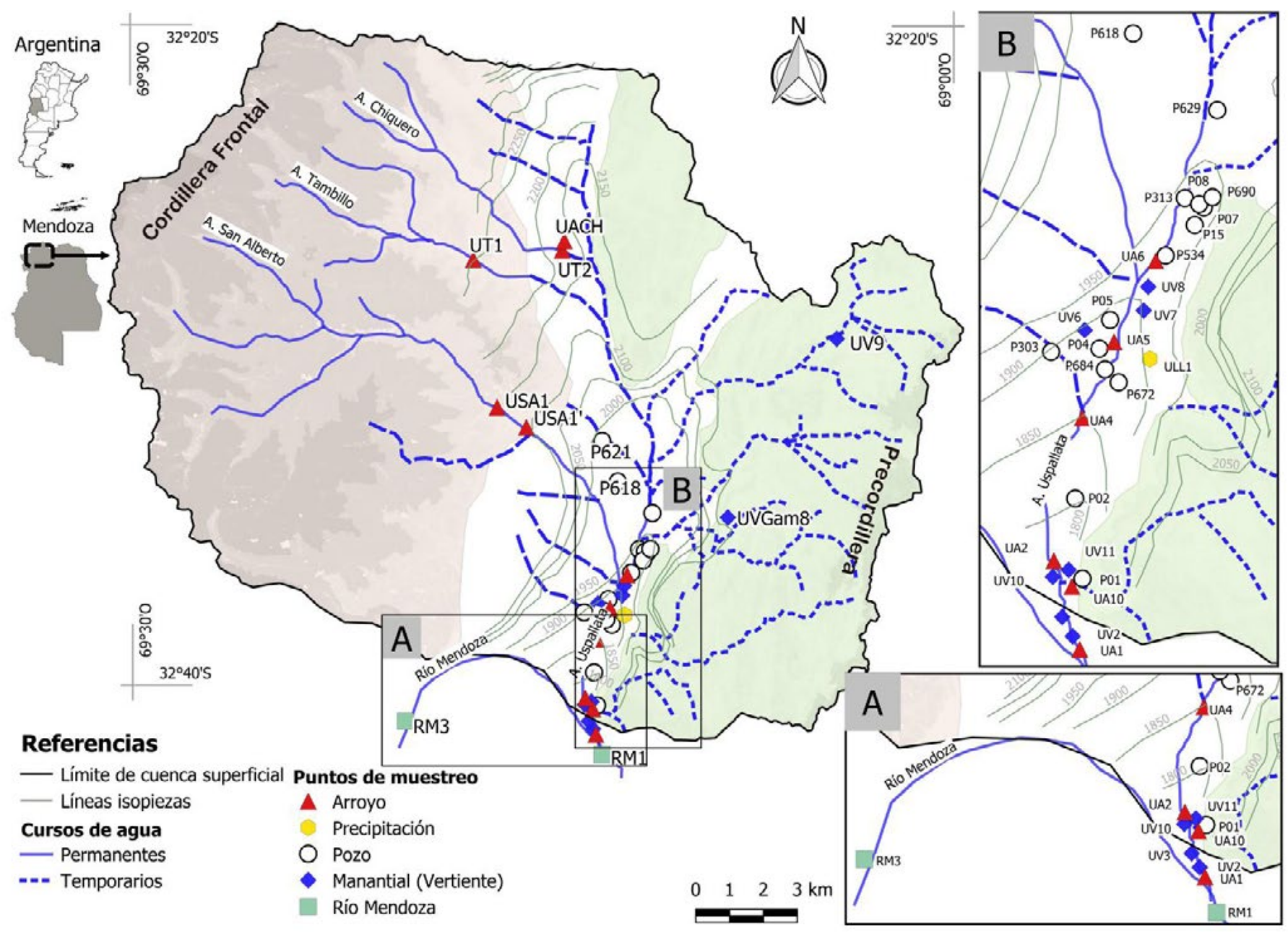

Figura 1. Izquierda: Mapa de la zona de estudio mostrando una piezometría regional tentativa y los sitios de muestreo. [A] Detalle del sector de descarga del A. Uspallata en el río Mendoza. [B] Detalle de la zona central del Valle de Uspallata.

Figure 1. Left: Map of the study area showing a tentative regional piezometry and the sampling sites. [A] Detail of the discharge sector of the Uspallata Creek into the Mendoza River. [B] Enlarged view of the central Uspallata Valley.

Todo el sistema hídrico del valle fluye hacia el sur y descarga al río Mendoza, principal recurso hídrico que abastece al oasis socio-productivo de mayor importancia de la provincia de Mendoza.

\section{Metodología}

Para identificar la procedencia del agua superficial y subterránea a lo largo del valle se estableció una red de monitoreo de 39 puntos compuesta por 13 puntos en arroyos permanentes, 9 manantiales (vertientes), 16 pozos y 1 punto de colección de lluvia (Fig. 1). Los pozos presentan profundidades variables, pero se pueden agrupar en pozos someros (entre 8 y $33 \mathrm{~m}$ ) y pozos profundos (entre 47 y $96 \mathrm{~m}$ ).

Se realizaron dos campañas de monitoreo hidroquímico, isotópico, piezométrico y de aforos en arroyos, una correspondiente al periodo de estiaje invernal de 2016 (agosto) y otra a la época de crecida de caudales en verano de 2017 (febrero). En el momento de escribir este trabajo solo se tenían datos de una muestra de lluvia local del periodo $16 / 11 / 16$ al 31/03/17. En cada punto de muestreo se midió en campo el pH, la conductividad eléctrica (CE) y la temperatura, utilizando un equipo multiparamétrico calibrado marca WTW. Para análisis fisicoquímico (iones mayoritarios, alcalinidad, $\mathrm{pH}$ y $\mathrm{CE}$ ) se tomó $1 \mathrm{~L}$ de agua en envases de tereftalato de polietileno (PET), manteniéndose las muestras refrigeradas a $4{ }^{\circ} \mathrm{C}$ aproximadamente durante la campaña y hasta su traslado al laboratorio de la FIUNCuyo. Para análisis isotópico $\left({ }^{2} \mathrm{H} \mathrm{y}{ }^{18} \mathrm{O}\right)$ se tomaron muestras de $125 \mathrm{~mL}$ en botellas PET, que fueron selladas con parafilm y analizadas en el laboratorio de hidrología isotópica del OIEA (Viena). Los resultados se expresan como desviaciones isotópicas $(\delta)$ en tanto por mil $(\%)$ relativas al patrón de agua oceánica de Viena (VSMOW) (Gonfiantini, 1978), aunque para aligerar el texto se ha omitido el nombre del patrón. Las incertidumbres son $\pm 0,1 \%$ para $\delta^{18} \mathrm{O} y \pm$ $0,5 \%$ para $\delta^{2} \mathrm{H}$. Los aforos fueron realizados en arroyos permanentes (Fig. 1) con molinete marca SIAP. 
Se trazó una piezometría regional tentativa de la cuenca del valle utilizando datos piezométricos de pozos someros, cotas de arroyos y nacientes y curvas de nivel.

Las características y el origen más probable de las aguas se estudiaron con apoyo del diagrama de Piper y comparando los datos isotópicos de ambas campañas con la recta meteórica mundial establecida por Craig (1961). El estudio del origen del agua a lo largo del valle se complementó con un corte hidrogeológico longitudinal para el cual se reinterpretaron SEVs realizados por el Instituto Nacional del Agua en la década de 1990 (Gianni y Vaca, 1994) y se incorporó información isotópica. La posible procedencia del agua subterránea en el sector central del valle se analizó con balances de masa de $\delta^{18} \mathrm{O}$ y $\delta^{2} \mathrm{H}$ (Ecuación 1; Mook, 1970) y con esa misma técnica se evaluó la descarga del Valle de Uspallata al río Mendoza.

$$
\Delta^{18} O_{\text {Mezcla }}=f_{1} \Delta{ }^{18} O_{\text {fuente1 }}+f_{2} \Delta^{18} O_{\text {fuente2 }}
$$

donde:

f1: proporción del componente 1 en la mezcla. f2: proporción del componente 2 en la mezcla

$$
f_{1}+f_{2}=1
$$

Estos cálculos no se pudieron contrastar con balances de masa de cloruro debido a la pequeña variabilidad de las concentraciones en las aguas. Además, la incertidumbre analítica de los datos de $\mathrm{Cl}^{-}\left( \pm 5 \mathrm{mg}^{-\mathrm{L}^{-1} \text {; }}\right.$ método argentométrico $4500 \mathrm{Cl}^{-}$) se encuentra dentro de las diferencias de concentración observadas entre las muestras usadas para el balance. No obstante, dado que los isótopos estables no son solutos sino componentes intrínsecos de la molécula de agua, los balances realizados son tan confiables como los balances de masa de agua.

\section{Resultados y discusión}

\section{Composición de las aguas y su posible origen}

Los acuíferos del Valle de Uspallata reciben aportes de ambas márgenes del valle y del sector norte de la cuenca, según lo determinado por la piezometría tentativa realizada (Fig. 1).

Las aguas de la cuenca del A. Uspallata (incluyendo arroyos, pozos y manantiales) muestran valores de CE de bajos a medios $\left(210-1840 \mu \mathrm{S} . \mathrm{cm}^{-1}\right.$ en agosto de $2016 ; 206-1745 \mu \mathrm{S} . \mathrm{cm}^{-1}$ en febrero de 2017 ) y valores de $\mathrm{pH}$ ligeramente alcalinos $(\approx 8)$. La mayoría de las aguas son de tipo bicarbonatado-cálcico y presentan poca variación estacional (Fig. 2), lo cual es consistente con el estudio de los antecedentes hidroquímicos de la zona recopilados por Lana et al. (2017). Se excluyen de esta generalidad los siguientes puntos: i) el manantial UVGam8 (Precordillera; Fig. 1), que pasó de ser $\mathrm{SO}_{4}-\mathrm{Ca}$ en invierno a $\mathrm{SO}_{4}-\mathrm{Na}$ en verano, y los pozos $\mathrm{P} 690$ y $\mathrm{P07}$ (margen oriental del A. Uspallata) y el manantial UV9 (Precordillera; Fig. 1), que tienen aguas $\mathrm{SO}_{4}-\mathrm{Na}$ en ambas estaciones. Las aguas bicarbonatadas-cálcicas se relacionan con flujos procedentes de la vertiente occidental de la cuenca (Cordillera Frontal). Las aguas sulfatadas-sódicas y cálcicas se relacionan con flujos procedentes de la vertiente oriental (Precordillera), cuya composición litológica difiere localmente de la de la parte occidental (Lana et al., 2017). ii) El manantial UV7 (margen oriental del A. Uspallata) y el pozo P04 (margen occidental) (Fig. 1) son de tipo $\mathrm{HCO}_{3}-\mathrm{Na}$, y aún se está por establecer su origen. iii) Finalmente, las aguas del río Mendoza son de tipo $\mathrm{SO}_{4}-\mathrm{Ca}$, lo que es coherente con la existencia de yesos en su cuenca.

En la Fig. 3 se presentan los valores de $\Delta^{18} \mathrm{O}$ y $\Delta^{2} \mathrm{H}$ de las muestras de ambas campañas. Como referencia se ha incluido el valor medio anual de la precipitación en la ciudad de Mendoza entre 1981 y 1999 (Dapeña, 2008) y el de una muestra de nieve de la cuenca del río Vallecitos (11/2013), ubicado en la Cordillera Frontal al sur de la zona de estudio (Sileo et al., 2015).

Todas las muestras se ajustan a la recta meteórica mundial, excepto UVGam y UV9, las cuales presentan un exceso de deuterio menor a 10 . El hecho de que ambos manantiales se encuentran en la Precordillera (donde domina la precipitación líquida), sumado a que los caudales de descarga son pequeños y a que durante el muestreo se observaron sales en el entorno de ambas surgencias, sugiere que son resultado de procesos de evaporación más que de sublimación (Fig. 3). Los contenidos isotópicos del conjunto de las muestras se encuentran entre -19 y $-6 \%$ para $\delta^{18} \mathrm{O}$ y entre -140 y $-40 \%$ para $\delta^{2} \mathrm{H}$. Estos amplios rangos son consistentes con la gran variación de la altitud en el valle (entre 1750 y $5000 \mathrm{~m} \mathrm{snm}$, aproximadamente) y también con las esperables diferentes proporciones de precipitación de fuentes de humedad provenientes del Atlántico y del Pacífico (Crespo et al., 2016). Las muestras del río Mendoza están isotópicamente empobrecidas respecto a las aguas del Valle de Uspallata, como es esperable por la mayor altitud de su cuenca (Panarello et al., 1993; Panarello y Dapeña, 1996; Massone et al., 2016).

La mayor parte de las aguas del Valle de Uspallata presenta una variación isotópica comprendida entre -16 y $-12 \%$ para $\delta^{18} \mathrm{O}$, mostrando un enriquecimiento isotópico de sur a norte. En este rango están también las aguas de los arroyos Chiquero, Tambillo y San Alberto, localizados en el sector de Cordillera Frontal. Las muestras P690, UV9 y UVGam, que proceden de puntos situados en la vertiente oriental del valle (sector de Precordillera), tienen composición isotópica enriquecida respecto a las demás, en un rango comprendido entre -11 y $-6 \%$ para $\delta^{18} \mathrm{O}$. Aunque sólo se contaba con una muestra de lluvia local (tomada en la parte baja del valle), la cercanía de esas muestras a la composición isótopica de esa lluvia sugiere un origen dominante en la recarga de la lluvia local para las aguas subterráneas del sector oriental, pero es necesario mejorar la información a este respecto. Por otro 


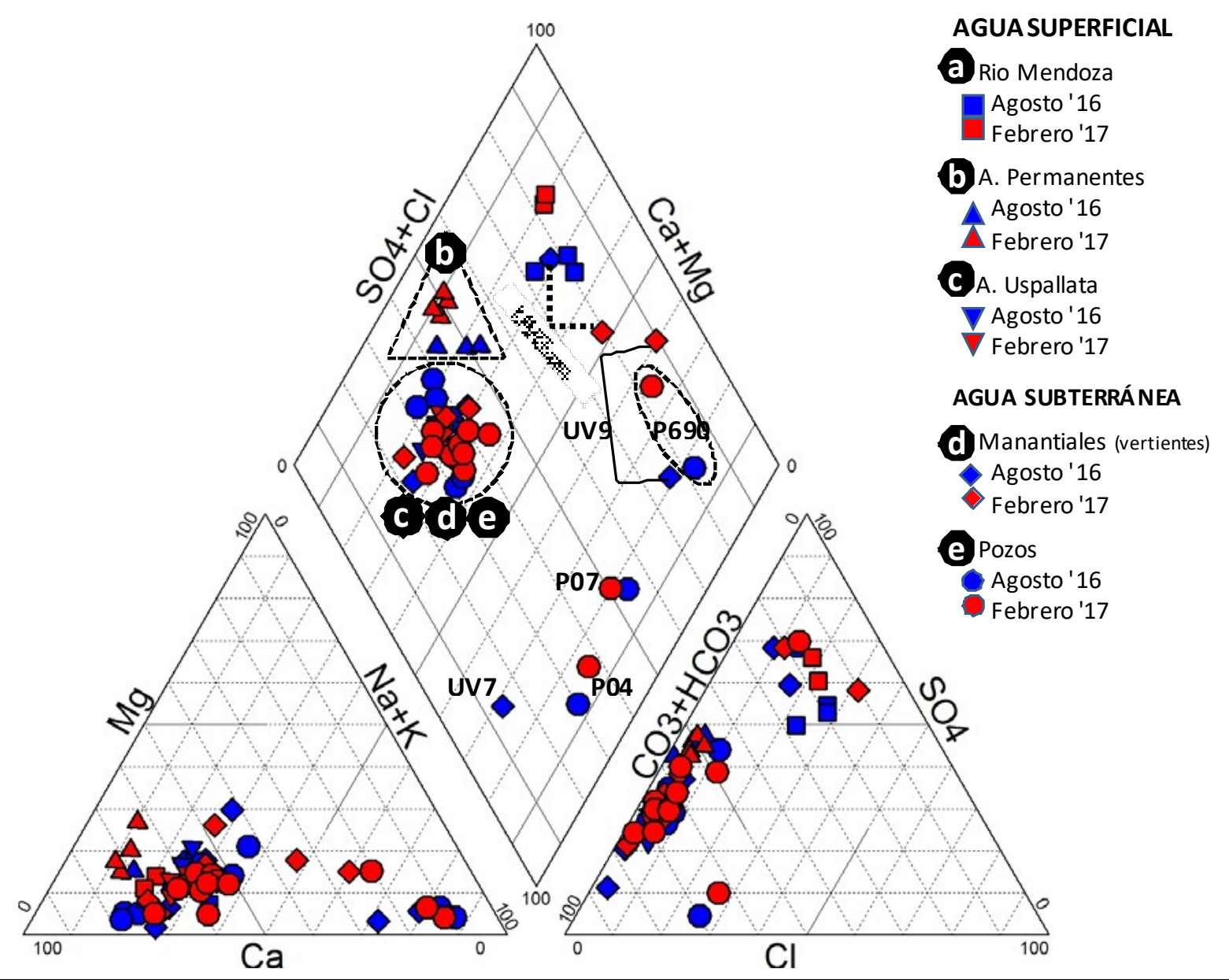

Figura 2. Diagrama de Piper de las muestras de las campañas de agosto 2016 (invierno) y febrero 2017 (verano). Figure 2. Piper diagram showing the samples of August 2016 (winter) and February 2017 (summer) campaigns.

lado, el hecho de que las aguas subterráneas (pozos y manantiales) de la mayor parte del valle estén en el rango de los arroyos cuyas nacientes se ubican en la Cordillera Frontal indica que la precipitación sobre esta cordillera es la principal fuente de recarga del acuífero.

\section{Estructura geológica y control de las entradas laterales}

El corte geológico longitudinal del valle (Fig. 4, izquierda) realizado con los SEV de Gianni y Vaca (1994) demostró que entre los sectores norte y central del valle existe un levantamiento del basamento neógeno que disminuye el espesor de sedimentos cuaternarios (Fig. 4, derecha). Analizando la distribución en profundidad de la CE y los valores de $\delta^{18} \mathrm{O}$ se observó que, del levantamiento del basamento hacia el sur, los valores de CE aumentan y los de $\delta^{18} \mathrm{O}$ son más empobrecidos que los correspondientes al sector norte. Esto se interpreta, a priori, como resultado de dos procesos que están teniendo lugar en los sectores centro y sur del valle (Fig. 4): 1) el incremento de la CE respondería al impacto que producen las actividades antrópicas desarrolladas en el valle sobre la química de las aguas. Este aumento de la concentración salina se evidencia no sólo en los pozos más someros (como el P02) sino también en las aguas superficiales, receptoras de los retornos de riego generados en el área. 2) El empobrecimiento isotópico se atribuye a una entrada de agua subterránea que, por su procedencia de cotas más altas y la mayor magnitud de su flujo, modificaría las características del agua del acuífero desde donde termina el levantamiento de la base del neógeno hasta donde se produce la descarga de todo el valle al río Mendoza. Este ingreso de agua más ligera podría tener lugar a través de discontinuidades del levantamiento de la base, o podría ocurrir desde el subálveo del A. San Alberto, o bien podría tener lugar mediante ambos mecanismos. Para evaluar la magnitud de esta entrada se realizó un balance de masa isotópico. Se seleccionaron tres pozos, teniendo en cuenta su ubicación en el área: dos sobre el levantamiento del basamento, uno representativo del agua recargada en el sector norte del acuífero (P534), otro representativo 
del agua más ligera que entra al acuífero por el sector central desde el oeste (P05) y otro representativo de la mezcla de las dos aguas anteriores (P303). EI balance de masa realizado (Tabla 1) indica que esta entrada de agua subterránea más ligera representaría, en invierno de 2016 (agosto), aproximadamente el $70 \%$ del flujo subterráneo que circula por esa área. Para corroborar la hipótesis del aporte diferenciado de agua subterránea en este sector se realizaron mediciones de caudal sobre el A. Uspallata. Con aforos diferenciales se observó que en esa zona el caudal del A. Uspallata se triplicaba, lo que apoya la hipótesis propuesta a partir de los datos isotópicos.

\section{Descarga del valle al río Mendoza}

Para estimar la descarga del sistema hidrológico del Valle de Uspallata al río Mendoza mediante balance de masas isotópico se consideraron dos puntos de muestreo sobre el río Mendoza, uno antes de entrar a la Pampa de Uspallata (RM3; Fig1A) y otro ubicado aguas abajo de la descarga del A. Uspallata (RM1, representativo de la mezcla del agua del río Mendoza y el agua del Valle de Uspallata) y se consideró el agua del punto UA1 como representativa del aporte del Valle de Uspallata. Considerando los valores de $\delta^{18} \mathrm{O}$ y $\delta^{2} \mathrm{H}$ se obtuvo que, durante la época de estiaje estudiada (agosto-2016), el aporte total del Valle de Uspallata al río Mendoza fue del $12-13 \%$ del caudal del río en RM1. En el punto RM1 se consideraron los valores de caudal medidos en la estación de aforos Guido. EI caudal medio diario medido en esta estación en agosto de 2016 fue de $19,9 \mathrm{~m}^{3} . \mathrm{s}^{-1}$, lo que supone que la salida total (superficial y subterránea) de agua desde el Valle de Uspallata al río Mendoza en esa época sería de aproximadamente $2,60 \mathrm{~m}^{3} \cdot \mathrm{s}^{-1}$ (Tabla 1). Realizando el balance con ambos isótopos, la contribución del sistema hidrológico de Uspallata al río Mendoza en verano de 2017 (febrero) habría sido del 3-6\% del caudal medio del río Mendoza en RM1 en ese mes. Considerando un caudal medio en ese punto de 84,64 $\mathrm{m}^{3} \cdot \mathrm{s}^{-1}$, la descarga total del sistema de Uspallata ese mes habría sido aproximadamente $2,9 \mathrm{~m}^{3} . \mathrm{s}^{-1}$.

Del análisis de la información interpretada se dedujo un modelo conceptual preliminar de la dinámica del acuífero del Valle de Uspallata, identificando cuatro sectores con características diferenciadas (Fig. 4, derecha):

(a) Sector Norte: se recarga principalmente con aguas procedentes de cotas menores a las de la cuenca del A. San Alberto, en principio consistentes con las de los arroyos Chiquero y Tambillo.

(b) Sector Centro: caracterizado por la recarga con agua que provendría de cotas altas, en principio similares o mayores a las de la cuenca alta del A. San Alberto.

(c) Sector Sur: caracterizado por mezcla de las aguas de los dos sectores anteriores y con un aporte de más del $70 \%$ de las aguas del Sector Centro en invierno.

(d) Sector de confluencia con el río Mendoza: la descarga hídrica del Valle de Uspallata se produce tanto por flujo superficial como en forma subterránea, y su magnitud relativa al caudal del río Mendoza parece ser más del doble en invierno que en verano. Esto hace que en invierno los aportes del Valle de Uspallata produzcan una ligera mejora de la calidad del agua

\begin{tabular}{|c|c|c|c|c|c|c|c|c|c|c|}
\hline \multirow[b]{2}{*}{ ID } & \multicolumn{2}{|c|}{$\Delta^{2} \mathrm{H}(\% \mathrm{o})$} & \multicolumn{2}{|c|}{$\%$ en la mezcla } & \multicolumn{2}{|c|}{$\Delta^{18} \mathrm{O}(\%)$} & \multicolumn{2}{|c|}{$\%$ en la mezcla } & \multicolumn{2}{|c|}{ Caudales $\left(\mathrm{m}^{3} \cdot \mathrm{s}^{-1}\right)$} \\
\hline & $\begin{array}{c}\text { Ago '16 } \\
\text { (1) }\end{array}$ & $\begin{array}{c}\text { Feb '17 } \\
\text { (2) }\end{array}$ & $\%(1)$ & $\%(2)$ & $\begin{array}{c}\text { Ago '16 } \\
\text { (1) }\end{array}$ & $\begin{array}{c}\text { Feb '17 } \\
\text { (2) }\end{array}$ & $\%(1)$ & $\%(2)$ & (1) & (2) \\
\hline P05 -fuente 1 & $-117,41$ & $-115,49$ & 73 & s.d. & $-16,08$ & $-15,64$ & 74 & s.d. & & \\
\hline $\begin{array}{l}\text { P534_ } \\
\text { fuente2 }\end{array}$ & $-99,51$ & $-99,06$ & 27 & s.d. & $-13,77$ & $-13,73$ & 26 & s.d. & & \\
\hline $\begin{array}{l}\text { P303_ } \\
\text { mezcla }\end{array}$ & $-112,56$ & s.d. & & & $-15,49$ & s.d. & & & & \\
\hline $\begin{array}{l}\text { UA1_- } \\
\text { fuente1 }\end{array}$ & $-108,05$ & $-105,56$ & 12 & 6 & $-14,53$ & $-14,43$ & 13 & 3 & $0,38^{b}$ & $1,8^{b}$ \\
\hline $\begin{array}{l}\mathrm{RM}_{3} \\
\text { fuente2 }\end{array}$ & $-142,36$ & $-141,48$ & 88 & 94 & $-18,85$ & $-18,90$ & 87 & 97 & s.d. & s.d. \\
\hline $\begin{array}{l}\mathrm{RM} 1 \_ \\
\text {mezcla }\end{array}$ & $-138,16$ & $-139,19$ & & & $-18,29$ & $-18,75$ & & & $19,9^{\mathrm{a}}$ & $84,64^{a}$ \\
\hline $\begin{array}{l}\text { (1) Agostc } \\
\text { s.d. Sin d } \\
\text { a. Caudale } \\
\text { b. Caudale }\end{array}$ & $\begin{array}{l}6 ;(2) \\
\text { forma } \\
\text { edide }\end{array}$ & $\begin{array}{l}\text { ero } 201 \\
\text { mor la es } \\
\text { moline }\end{array}$ & drc & $G$ & & & & & & \\
\hline
\end{tabular}

Tabla 1. Datos isotópicos de los sitios de muestreo empleados para el cálculo de mezclas y caudales en el A. Uspallata y el río Mendoza. Table 1. Isotopic data of the sampling sites used to calculate mixtures and flow measured in the Uspallata Creek and the Mendoza River. 


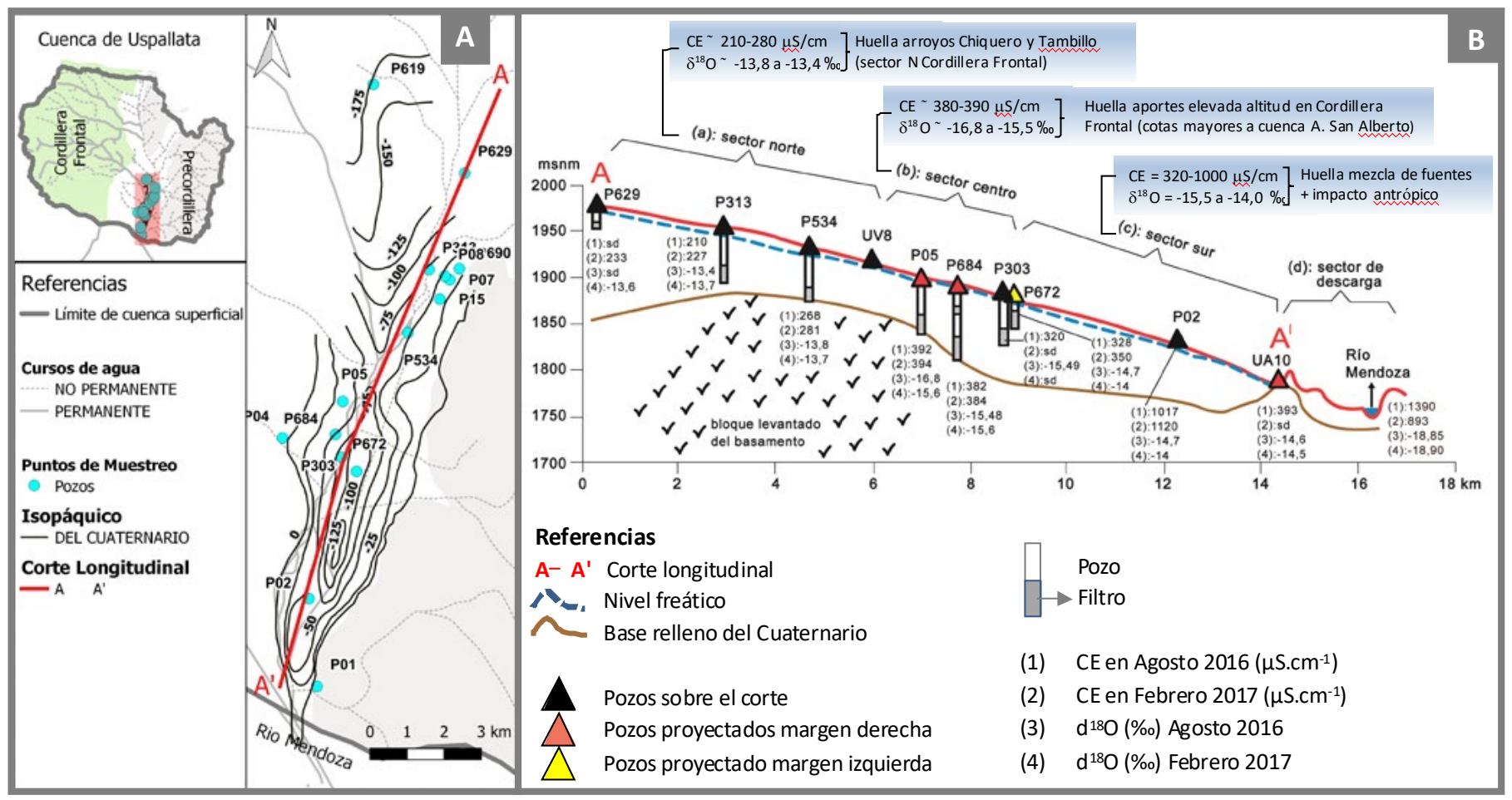

Figura 4. Modelo conceptual preliminar del acuífero del Valle de Uspallata. [A] Mapa isopáquico del relleno cuaternario, posición de los pozos de muestreo y traza de corte longitudinal. [B] Corte longitudinal del valle con posición relativa de los pozos y sus correspondientes valores de conductividad eléctrica (CE) y $\delta^{18} \mathrm{O}$ en Agosto 2016 (invierno) y Febrero 2017 (verano).

Figure 4. Preliminary conceptual model of the Uspallata aquifer. [A] Isopach map of the Quaternary, wells position and plot of the longitudinal section. [B] Longitudinal section of the valley with the relative position of the wells and their electrical conductivity and $\delta^{18} \mathrm{O}$ in the August 2016 (winter) and February 2017 (summer) campaigns.

del río Mendoza, evidenciada por una pequeña disminución de su salinidad y un ligero enriquecimiento isotópico. En verano, en cambio, el mayor caudal del río Mendoza amortigua el impacto de los aportes del Valle de Uspallata y, si bien se observa una pequeña variación de la composición isotópica, no se evidencia una disminución de la salinidad.

\section{Conclusiones}

Aunque los cálculos presentados en este trabajo son preliminares y se están comprobando con otros trazadores, la contrastada fiabilidad de la técnica utilizada les proporciona una notable confianza.

El modelo conceptual preliminar obtenido sobre la procedencia del agua que recarga el acuífero del Valle de Uspallata y sobre la contribución de la descarga del Valle al río Mendoza debería ser la base para diseñar una red de monitoreo que permita una mejor gestión del recurso. La red de control debería tener en cuenta los siguientes aspectos: (a) De los cuatro sectores diferenciados, el Sector Norte es el que posee mejor calidad de agua, pero el volumen aportado al conjunto es menor que el Sector Centro. Futuros estudios deberán estar orientados a identificar sus fuentes a fin de monitorear su evolución quí- mica y asegurar su protección, puesto que en este sitio se localizan dos de los tres pozos que abastecen de agua potable a la Villa de Uspallata. (b) El Sector Centro es donde parece tener lugar la mayor parte de la recarga del acuífero de Valle de Uspallata, pero sus aguas son más mineralizadas que las del Sector Norte. (c) El Sector Sur merece especial atención para asegurar la sustentabilidad del recurso hídri$\mathrm{co}$, pues los pozos más superficiales tienen una CE más elevada que los más profundos, posiblemente debido al impacto de las actividades agrícolas que se desarrollan en la zona. Se debe evitar que esta afección se propague a todo el espesor del acuífero. (d) El sector donde se produce la descarga al río Mendoza está bien localizado, por lo que es relativamente fácil realizar monitoreos que informen de la evolución temporal de la descarga total del sistema al río Mendoza y de su efecto sobre la cantidad y la calidad del agua de este.

\section{Agradecimientos}

Los autores agradecen a los tres revisores anónimos sus observaciones y contribuciones a la mejora de este trabajo. 


\section{Referencias}

Caminos, R. 1979. Cordillera Frontal, en Geología Regional Argentina, Vol I. Academia Nacional de Ciencias, Córdoba. Argentina.

Capitanelli, R. 1972. Geomorfología y clima de la Provincia de Mendoza. X Jornadas Argentinas de Botánica. Las Ciencias Naturales en la Provincia de Mendoza. Bol. Soc. Argent. Bot 13. Suplemento

Cortés, J. M; González Bonorino, G.; Koukharsky, M. M. L; Pereyra, F. X. y Brodtkorb, A. 1997. Memoria Hoja Geológica 3369-09 - Subsecretaría de Minería de la Nación. Servicio Geológico Minero Argentino. Buenos Aires. Argentina.

Craig, H. 1961. Isotope variations in meteoric waters. Science, 133: 1702-1703.

Crespo, S., Aranibar, J., Gómez, L., Schwikowski, M., Bruetsch, S., Cara, L. y Villalba, R. 2016. Ionic and stable isotope chemistry as indicators of water sources to the Upper Mendoza River basin, Central Andes of Argentina. Hydrological Sciences Journal 62(4): 588-605.

Dapeña, C. 2008. Isótopos Ambientales Livianos: su aplicación en hidrología e hidrogeología. PhD Tesis, Universidad de Buenos Aires.

Gianni, R. y Vaca, A. 1994. Investigación geofísica preliminar en el sector Sur de la Cuenca de Uspallata. INA-CRAS- IT - 153.

Gonfiantini, R. 1978. Standards for stable isotope measurements in natural compounds. Nature 271: 534-536.

INDEC. 1991. Censo Nacional de Población y vivienda 1991. Buenos Aires. Argentina.

INDEC. 2010. Censo Nacional de Población y vivienda 2010. Buenos Aires. Argentina

Lana, B.; Rovira, S.; Gómez, M.L.; Tognoli, P., Bueno, P. y Ibáñez, S. 2017. Análisis hidroquímico de las aguas superficiales y subterránea del valle de Us-
pallata-Mendoza (1970-2015). XX Congreso Geológico Argentino, Tucumán, Argentina.

Massone, H., Martínez, D., Vich, A., Quiróz Londoño, M., Trombotto, D., Grondona, S. 2016. Snowmelt contribution to the sustainability of the irrigated Mendoza's Oasis, Argentina: an isotope study. Environ Earth Sci 75:520

Mook, W.G. 1970. Stable carbon and oxygen isotopes of natural waters in the Netherlands. In: Proc. Isotope Hydrology, IAEA, Vienna: 163-189.

Panarello, H. y Dapeña, C. 1996. Mecanismos de recarga y salinización en las Cuencas de los ríos Mendoza yTunuyán, República Argentina. XII Congreso Geológico de Bolivia Actas 2:1-18.

Panarello, H.; Dapeña, C.; García, E. y Álvarez, A. 1993. Estudio isotópico e hidroquímico de los acuíferos de la Cuenca Norte mendocina, ríos Mendoza yTunuyan. XII Congreso Geológico Argentino, Actas VI: 197-207. Mendoza

Salomón, M.; Soria, N.; Fernández, R. y Rubio, C. 2005. Evaluación de degradación de tierras en zonas áridas: sitio piloto Mendoza, Departamento de Lavalle. Informe Técnico. LADyOT - IADIZA - CRICYT. Mendoza, Argentina.

SEGEMAR. 1997. Hoja Geológica 3369-09, Uspallata. Servicio Geológico Minero Argentino.

Sileo, N., Trombotto, D. y Dapeña, C. 2015. Estudios preliminares del agua, nieve y hielo en la cuenca del río Vallecitos, Mendoza, Argentina. Acta Geológica Lilloana, 27. 130 pp.

Vaca, A. 1985. Descripción del Mapa Hidrogeológico Preliminar del Valle de Uspallata. Mendoza. DI166. San Juan. Argentina.

Vaca, A. 1993. Recursos hídricos subterráneos y aguas termales. En: Geología y Recursos Naturales de Mendoza. Eds. Pazos, J. A., Bessone, J. L., Vivas J. A., Vaca, A., Wetten, C. y Valero, C. E. Relatorio XII Congr. Geol. Arg: 551-599.
Recibido: julio 2019

Revisado: noviembre 2019

Aceptado: enero 2020

Publicado: marzo 2021 